\title{
Synchronized Intermittent Mandatory Ventilation: Time to Send This Workhorse Out to Pasture
}

After its development as a weaning modality in the early $1970 s,{ }^{1}$ intermittent mandatory ventilation (IMV) quickly became a very popular mode of ventilation. Continuous mandatory ventilation, the prevailing mode in use prior to the introduction of IMV, permitted the patient to determine the number of ventilator breaths by sensing patient inspiratory effort. In contrast, IMV mode delivered a set number of controlled mandatory breaths while allowing the ventilated patient to breathe spontaneously (and unassisted) between mandatory breaths. Weaning was to occur by slowly reducing the set ventilator rate, allowing the patient to gradually take over the work of breathing. ${ }^{1}$ To overcome inherent asynchrony with IMV, a synchronized mode known as synchronized intermittent mandatory ventilation (SIMV) was developed, which senses patient inspiratory effort and enables the patient to receive "synchronized" patient-triggered mandatory breaths up to the set rate.

SIMV quickly became the preferred mode in many ICUs, for both ventilation and weaning, in the overwhelming majority of institutions. ${ }^{2}$ However, enthusiasm for SIMV subsequently waned as physiologic studies examining its effects on ventilated patients questioned its theoretical benefits. Hudson et $\mathrm{al}^{3}$ showed that IMV may be helpful in correcting abnormally high $\mathrm{pH}$ in patients with respiratory alkalosis, but at the cost of increased work of breathing. Subsequently, both Marini et $\mathrm{al}^{4}$ and Imsand et $\mathrm{al}^{5}$ showed that work of breathing during SIMV was determined by the work of the spontaneous breaths, and that the mandatory delivered breaths did not effectively unload the respiratory muscles in critically ill patients with respiratory failure. Those authors concluded that neurologic control of the respiratory muscles, and hence respiratory muscle work, was not adaptable on a breath-by-breath basis, as is purported to occur by initial supporters of SIMV. The addition of pressure support for the spontaneous patient breaths to the mandatory patient-triggered breaths was subsequently found to decrease the work of breathing during SIMV,${ }^{6}$ and use of SIMV with pressure support (SIMVPS) ventilation became more widespread.

Henceforth, SIMV developed both ardent supporters and detractors. While experts debated the physiologic advantages and disadvantages of SIMV, randomized controlled trials were initiated to compare clinical outcomes of the various ventilator modes. Esteban et $\mathrm{al}^{7}$ and Brochard et $\mathrm{al}^{8}$ both showed that SIMV, as a weaning strategy, was inferior to either pressure support or T-piece trials, with SIMV on average slowing the weaning process from mechanical ventilation by $2-4$ days. Thus, over time, the percentage of patients being weaned via SIMV or SIMV-PS declined from over $90 \%$ in the 1980 s to just under $18 \%$ in $2004 .{ }^{9}$ More recently, in a large multinational observational study examining thousands of patients, Ortiz et al ${ }^{10}$ found that, compared to continuous mandatory ventilation, SIMV-PS was less likely to be used in Latin America or Europe, and more likely to be used in North America, and in patients with lower severity of illness scores, as well as those ventilated postoperatively or for trauma.

While use of the SIMV mode for weaning declined, it also became clear that patient-ventilator asynchrony is associated with adverse outcomes, including a longer duration of mechanical ventilation. Studies by Chao et al ${ }^{11}$ and Thille et al ${ }^{12}$ clearly established that link, and suggested that elimination of asynchrony can also facilitate the process of liberation from mechanical ventilation. However, while the trials examining patient-ventilator asynchrony have examined mainly patients in medical ICUs, where the continuous mandatory ventilation mode predominates, patients in surgical ICUs are more likely to be ventilated via SIMV or SIMV-PS. Considering that patient populations differ substantially between the different types of ICUs, studies examining the occurrence and clinical impact of asynchrony in non-medical ICUs are of interest.

See the Original Study on Page 1847

In this light, the study by Robinson et $\mathrm{al}^{13}$ in this issue of RESPIRATORY CARE investigates the incidence and types of asynchrony specifically seen in trauma patients in a surgical ICU. Robinson et al examined 30 min recordings of ventilator waveforms in 35 traumatically injured patients admitted to a single institution. They tabulated asynchronous breaths, which included ineffective triggering, double-triggering, short- and long-cycle breath asynchrony, and breath stacking. In addition to having a lower mortality and shorter hospital stay, the patient population was younger, healthier, and had a lower incidence of COPD than have other studies of asynchrony in medical ICUs. 


\section{Synchronized Intermittent Mandatory Ventilation}

Robinson et al found that asynchronous patients, defined as those with an asynchrony index (asynchronous breaths/ total breaths) $\geq 10 \%$, constituted $25.7 \%$ of their population. The asynchronous subjects did not differ from the synchronous subjects with regard to demographic features, arterial blood gas values, or use of sedation. Moreover, there were no differences in ventilator or ICU days, hospital stay, or mortality, although there was a nonsignificant trend toward higher mortality and fewer subjects discharged home in the asynchronous group. The most striking finding was that SIMV-PS was used in all the asynchronous subjects, whereas only $38.5 \%$ of the non-asynchronous subjects were ventilated with SIMV-PS, making SIMV the mode most associated with asynchrony in this population.

Explanations for the greater asynchrony associated with SIMV-PS include the possibility that characteristics of the SIMV mode promote asynchrony, or that providers tend to prefer SIMV mode in asynchronous subjects. The latter possibility cannot be ascertained from the Robinson et al study, but seems unlikely. So how could SIMV predispose to asynchrony? Among the asynchronies Robinson et al recorded, failure to trigger occurs commonly with many modes, including pressure support ventilation when it is associated with a higher pressure support level, where the greater tidal volume may depress the effort on some breaths, causing failure to reach the trigger threshold. Double triggering is also commonly seen with many modes, especially volume-limited continuous mandatory ventilation, when the patient takes a large breath that exceeds the set tidal volume, precipitating triggering of a second ventilator breath during the same patient inhalation. However, both of these forms of asynchrony were, as a proportion, more often seen in the synchronous subjects and were not associated with use of SIMV-PS. Short-cycling, the third type of asynchrony recorded by Robinson et al, occurred occasionally during pressure support ventilation (as per the example given by Robinson et al) and did not occur in the asynchronous group.

The main cause of asynchrony in the asynchronous subjects (responsible for $54.7 \%$ of the asynchronies observed) was long-cycling. This also appears to have occurred during spontaneous pressure support breaths (as per the example given). It is unclear why this asynchrony occurred so frequently during SIMV-PS, because the average subject in the asynchronous group received 12 SIMV breaths and only 4 spontaneous pressure supported breaths minute. Perhaps the delivered tidal volume during SIMV breaths was sensed as low by subjects, leading them to take larger tidal volumes during the subsequent spontaneous breaths. Robinson et al also described "a unique asynchronous breath type" associated with SIMV, which consisted of breath stacking, and appears, from the example given, to consist of a mandatory breath delivered on top of a pressure support breath. This appears to be related to a fault in the
SIMV algorithm in the ventilator they used, and should prompt the manufacturer to correct it.

One of the important findings in the Robinson et al trial was that a higher asynchrony index was not associated with worse clinical outcomes: a finding unlike other studies of asynchrony. ${ }^{11,12}$ However, this has to be interpreted in light of the fact that the long-cycled breaths that were responsible for most of the asynchronies in the asynchronous group have not been associated with worse outcomes in previous studies. For example, the studies by Chao et al ${ }^{11}$ and Thille et al, ${ }^{12}$ which showed a significant association between asynchrony and poor outcomes, excluded both short- and long-cycle asynchronies from the analysis. Hence, the importance of long-cycle asynchrony is unknown, as it may represent a physiologic sigh to open atelectatic alveoli, or perhaps a physiologic response to perceived small SIMV breaths, as pointed out above, and may not have been reflective of cardiopulmonary pathology. In fact, when Robinson et al excluded short- and long-cycle asynchronies from their analysis, the association between asynchrony and SIMV seemed to dissipate. However, there was a trend toward greater mortality in the asynchronous subjects, though it did not approach statistical significance. That raises concern about beta error, given the small sample size. Ultimately, the relatively younger and healthier patient population in the Robinson et al study, compared to medical ICU studies on asynchrony, may have predisposed to relatively good outcomes, regardless of the ventilation mode used or associated asynchronies.

So what does all this mean with regard to SIMV? For patients requiring substantial ventilatory support there appears to be little advantage in applying SIMV-PS, as this requires selecting the appropriate ventilator rate and being prepared to make frequent adjustments in the level of pressure support provided to unstable patients with rapidly changing ventilation needs and lung mechanics. Continuous mandatory ventilation is a preferable mode, as it reduces the risk of providing an inappropriate level of ventilatory support, but instead allows the patient to determine the breathing frequency as metabolic demand shifts. For improving patients who are ready to be weaned or liberated from mechanical ventilation, SIMV-PS has similarly lost its appeal. The earlier studies by Marini et $\mathrm{al}^{4}$ and Imsand et $\mathrm{al}^{5}$ demonstrating that SIMV fails to gradually off-load the work of breathing as ventilator support is reduced, as it was initially conceived to do, certainly stemmed enthusiasm. And Ortiz et ${ }^{10}$ al showed that those initially ventilated with SIMV are more likely to be weaned using that same mode, to the detriment of patients, as demonstrated clearly by Brochard et $\mathrm{al}^{8}$ and Esteban et $\mathrm{al}^{7}$ in the weaning trials referenced above. Now, Robinson et al identify another disadvantage of SIMV: that it is associated with ventilator asynchrony. Although there were no other adverse clinical outcomes associated with its use, 


\section{Synchronized Intermittent Mandatory Ventilation}

these could certainly occur in a sicker less stable patient population.

Thus, SIMV should not be the initial mode of ventilation in critically ill patients, nor is it a desirable mode for weaning compared to pressure support ventilation or Tpiece trials. One might ask, why has the use of SIMV-PS persisted as a popular mode, especially in postoperative and anesthesia settings? Tradition and familiarity are undoubtedly important factors, and for relatively healthy populations lacking important cardiopulmonary or neurologic comorbidities, many different modes could be used successfully, including SIMV-PS, because these patients generally require little ventilatory support, for brief periods, and they wean and extubate without difficulty. But one wonders how much negative evidence one needs before sounding the death knell for a medical technology? Especially in older critically ill populations with multiple comorbidities, as seen in a typical medical ICU, asynchrony could adversely affect outcomes. Despite the study's limitations, the findings from Robinson et al, added to the previous negative studies, should make us think twice about using SIMV. While SIMV has been a workhorse in many an ICU, it is time to send this workhorse out to pasture.

Maher Ghamloush MD Nicholas S Hill MD Division of Pulmonary, Critical Care, and Sleep Medicine Department of Medicine Tufts Medical Center Boston, Massachusetts

Dr Ghamloush has disclosed no conflicts of interest. Dr Hill has disclosed relationships with Breathe Technologies, Respironics, and Fisher \& Paykel.

Correspondence: Maher Ghamloush MD, Pulmonary, Critical Care and Sleep Division, Tufts Medical Center, Tufts University School of Medicine, 800 Washington Street, Box 257 Boston MA 02111-1526. E-mail: mghamloush@tuftsmedicalcenter.org.

DOI: $10.4187 /$ respcare.02880

\section{REFERENCES}

1. Downs JB, Klein J, Desautels D, Modell JH, Kirby RR. Intermittent mandatory ventilation: a new approach to weaning patients from mechanical ventilators. Chest 1973;64(3):331-335.

2. Venus B, Smith RA, Mathru M. National survey of methods and criteria used for weaning from mechanical ventilation. Crit Care Med 1987;15(5):530-533.

3. Hudson LD, Hurlow RS, Craig KC, Pierson DJ. Does intermittent mandatory ventilation correct respiratory alkalosis in patients receiving assisted mechanical ventilation? Am Rev Respir Dis 1985;132(5): 1071-1074.

4. Marini JJ, Smith TC, Lamb VJ. External work output and force generation during synchronized intermittent mechanical ventilation. Effect of machine assistance on breathing effort. Am Rev Respir Dis 1988;138(5):1169-1179.

5. Imsand C, Feihl F, Perret C, Fitting JW. Regulation of inspiratory neuromuscular output during synchronized intermittent mechanical ventilation. Anesthesiology 1994;80(1):13-22.

6. Leung P, Jubran A, Tobin M. Comparison of assisted ventilator modes on triggering, patient effort, and dyspnea. Am J Respir Crit Care Med 1997;155(6):1940-1948.

7. Esteban A, Frutos F, Tobin MJ, Alía I, Solsona JF, Valverdú I, et al. A comparison of four methods of weaning patients from mechanical ventilation. N Engl J Med 1995;332(6):345-350.

8. Brochard L, Rauss A, Benito S, Conti G, Mancebo J, Rekik N, et al. Comparison of three methods of gradual withdrawal from ventilatory support during weaning from mechanical ventilation. Am J Respir Crit Care Med 1994;150(4):896-903.

9. Esteban A, Ferguson ND, Meade MO, Frutos-Vivar F, Apezteguía C, Brochard L, et al; VENTILA Group. Evolution of mechanical ventilation in response to clinical research. Am J Respir Crit Care Med 2008;177(2):170-177.

10. Ortiz G, Frutos-Vivar F, Ferguson ND, Esteban A, Raymondos K, Apezteguía C, et al; VENTILA Group. Outcomes of patients ventilated with synchronized intermittent mandatory ventilation with pressure support. Chest 2010;137(6):1265-1277.

11. Chao DC, Scheinhorn DJ, Stearn-Hassenpflug M. Patient-ventilator trigger asynchrony in prolonged mechanical ventilation. Chest 1997; 112(6): 1592-1599.

12. Thille AW, Rodriguez P, Cabello B, Lellouche F, Brochard L. Patient-ventilator asynchrony during assisted mechanical ventilation. Intensive Care Med 2006;32(10):1515-1522.

13. Robinson BR, Blakeman T, Toth P, Hanseman DJ, Mueller E, Branson RD. Patient-ventilator asynchrony in a traumatically injured population. Respir Care 2013;58(11):1847-1855. 